\title{
Direct Determination of Supermassive Black Hole Properties with Gravitational-Wave Radiation from Surrounding Stellar-Mass Black Hole Binaries
}

\author{
Hang Yu॰* and Yanbei Chen \\ TAPIR, Walter Burke Institute for Theoretical Physics, Mailcode 350-17 California Institute of Technology, \\ Pasadena, California 91125, USA
}

(Received 8 September 2020; revised 27 October 2020; accepted 14 December 2020; published 11 January 2021)

\begin{abstract}
A significant number of stellar-mass black-hole (BH) binaries may merge in galactic nuclei or in the surrounding gas disks. With purposed space-borne gravitational-wave observatories, we may use such a binary as a signal carrier to probe modulations induced by a central supermassive BH (SMBH), which further allows us to place constraints on the SMBH's properties. We show in particular the de Sitter precession of the inner stellar-mass binary's orbital angular momentum (AM) around the AM of the outer orbit will be detectable if the precession period is comparable to the duration of observation, typically a few years. Once detected, the precession can be combined with the Doppler shift arising from the outer orbital motion to determine the mass of the SMBH and the outer orbital separation individually and each with percent-level accuracy. If we further assume a joint detection by space-borne and ground-based detectors, the detectability threshold could be extended to a precession period of $\sim 100 \mathrm{yr}$.
\end{abstract}

DOI: 10.1103/PhysRevLett.126.021101

Introduction.-A significant number of stellar-mass binary black holes (BHs) detectable by LIGO [1] and Virgo [2] may merge in the vicinity of supermassive BHs (SMBHs) due to both dynamical interactions [3-9] and gaseous effects if accretion disks are present [10-17]. This possibility is strengthened as the Zwicky Transient Facility $[18,19]$ detected a potential electromagnetic counterpart [20] to the LIGO-Virgo event GW190521 [21,22], consistent with a binary $\mathrm{BH}$ merger in the accretion disk of an active galactic nucleus (AGN).

Beyond ground-based detectors, multiple space-borne gravitational-wave $(\mathrm{GW})$ observatories have been planned or conceived for the coming decades, including LISA [23], TianQin [24], Taiji [25], B-DECIGO [26,27], Decihertz Observatories [28], and TianGO [29]. Their sensitivities cover the $0.001-1 \mathrm{~Hz}$ band where a typical stellar-mass BH binary stays in the band for years. It thus opens up the possibility of using a stellar-mass BH binary as a carrier to probe modulations induced by a tertiary perturber which, as argued above, can be a SMBH in many cases. This is in analog to how pulsars are used to test the strong-field relativity [30] and it offers a complementary way to probe $\mathrm{SMBH}$ properties to extreme and very extreme mass-ratio inspirals (EMRI and X-MRI) [31-34].

The leading-order modulation is a Doppler shift due to the inner binary's orbital motion around the SMBH [35], creating frequency sidebands at $\Omega_{o}=\left(M_{3} / a_{o}^{3}\right)^{1 / 2}$ with $M_{3}$ the mass of the SMBH and $a_{o}$ the semimajor axis of the outer orbit. The extra dephasing of this effect can be determined up to

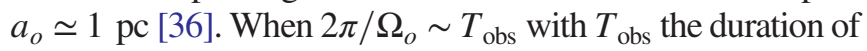
observation, $\Omega_{o}$ can be further resolved to constrain the mass density enclosed by the outer orbit [37].
In this Letter, we extend the field by including higherorder effects. The most significant one is that the inner orbital angular momentum (AM) $\boldsymbol{L}_{i}$ will experience a de Sitter-like (dS) precession around the outer AM $\boldsymbol{L}_{o}$ whose secular effect is [38-40]

$$
\frac{d \hat{\boldsymbol{L}}_{i}}{d t}=\Omega_{\mathrm{dS}} \hat{\boldsymbol{L}}_{o} \times \hat{\boldsymbol{L}}_{i}=\frac{3}{2} \frac{M_{3}}{a_{o}\left(1-e_{o}^{2}\right)} \Omega_{o} \hat{\boldsymbol{L}}_{o} \times \hat{\boldsymbol{L}}_{i},
$$

where $e_{o}$ is the eccentricity of the outer orbit. Here we have used the hat symbol to indicate unity vectors. As the binary precesses, the waveform undergoes both amplitude and phase modulations, thereby allowing the extraction of the precession signatures.

We illustrate the periods of the dS precession in the $\left(M_{3}, a_{o}\right)$ space in Fig. 1 with brown traces. The upper panel assumes a circular outer binary and the lower one has $e_{o}=0.9$. The solid (dashed) traces correspond to $P_{\mathrm{dS}}=2 \pi / \Omega_{\mathrm{dS}}=100(10) \mathrm{yr}$. As we will see later, these periods are the approximate detectability thresholds assuming a detection of a source $1 \mathrm{Gpc}$ away performed jointly by space-borne and ground-based detectors and by a TianGOlike detector alone. Also shown are the periods of the outer orbit (grey traces) and subleading corrections due to the Lense-Thirring precession (olive traces) and the LidovKozai effect (i.e., the Newtonian tidal effect; cyan traces). Explicit expressions are provided in the Supplemental Material [41].

To connect to astrophysical formation mechanisms of the inner binary, we indicate in dotted-orange lines the expected locations of migration traps in accretion disks [43] where 


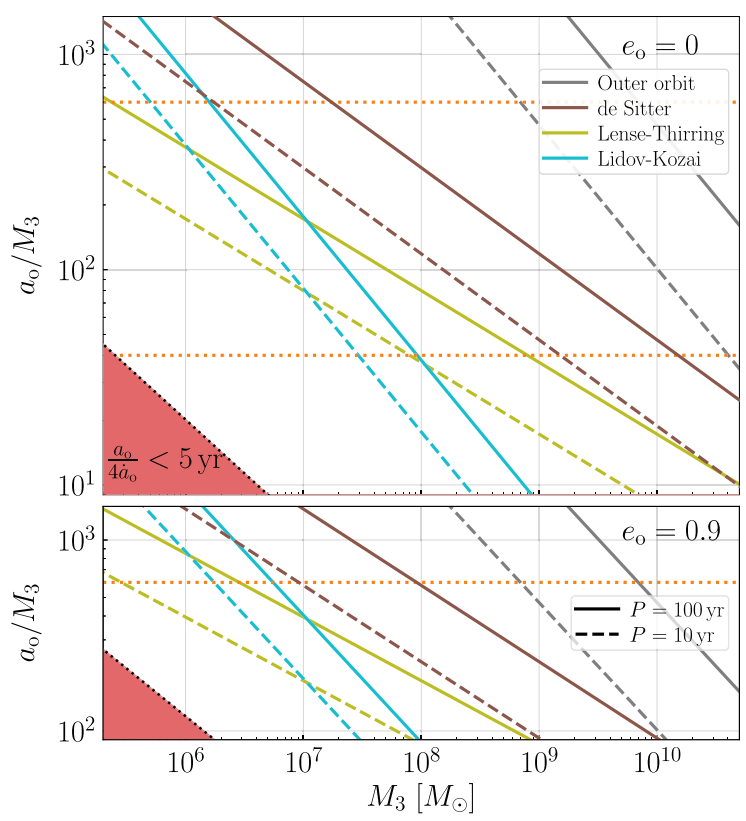

FIG. 1. Periods of various dynamical processes. Approximately, below the solid trace an effect is detectable with joint detection by space-borne and ground-based detectors. Below the dashed trace, the effect can be constrained with a single TianGO-like detector. We set the lower $y$ limit in each panel to $a_{o}\left(1-e_{o}\right)=9 M_{3}$. The decay of the outer orbit and hence the shaded region with $a_{o} / 4 \dot{a}_{o}<5$ yr is discarded. The triple stability [42] is always satisfied in the upper panel and its boundary is similar to the shaded region in the lower one.

massive objects are likely to accumulate and binaries may frequently merge. We find $P_{\mathrm{dS}}<100 \mathrm{yr}(10 \mathrm{yr})$ at the migration trap at $\simeq 600 M_{3}$ if $M_{3} \lesssim 2 \times 10^{7} M_{\odot}(2 \times$ $\left.10^{6} M_{\odot}\right)$ and the outer orbit is circular. When the outer orbit is eccentric, the $P_{\mathrm{dS}}<100 \mathrm{yr}$ boundary could be extended to further include $M_{3} \simeq 10^{8} \mathrm{yr}$.

For bare nuclei, binaries can also be produced by various dynamical processes. Studies suggest a detection rate of $\mathcal{O}(10-100) \mathrm{yr}^{-1} \mathrm{BH}$ binaries produced in the $a_{o} \lesssim 0.1 \mathrm{pc}$ region by the interaction channel $[3,5,6,44]$. Assuming a density profile $\propto a_{o}^{-2}$ [3], it indicates $\mathcal{O}(0.1-1)$ detection per year in the central $0.001 \mathrm{pc}$ region $\left(\simeq 200 M_{3}\right.$ for $M_{3}=10^{8} M_{\odot}$ ) where the dS precession could be significant. In fact, a binary formed in this channel may be launched to an outer orbit with significant eccentricity that reduces $P_{\mathrm{dS}}$ by a factor $\left(1-e_{o}^{2}\right)$ and allows the binary formed at greater $a_{o}$ to also experience significant precession (see the bottom panel of Fig. 1).

Once observed, the $\mathrm{dS}$ precession allows a direct determination of properties of the SMBH and the outer orbit. Note its rate is $\Omega_{\mathrm{dS}} / \Omega_{o}=M_{3} /\left[a_{o}\left(1-e_{o}^{2}\right)\right]$. When combined with the outer orbit's Doppler shift which tells us $\Omega_{o}=\sqrt{M_{3} / a_{o}^{3}}$ (and $e_{o}$ for elliptical orbits as we illustrate in the Supplemental Material [41] which includes Ref. [45]), we can therefore infer the values of $M_{3}, a_{o}$, and $e_{o}$ individually.

Before this method, there were two common approaches to directly determine the mass of an SMBH with a typical accuracy of tens of percent, either through directly observing the dynamics of the star or gas around the SMBH, or through reverberation mapping of the continuum emission of AGNs [46]. The former is limited to a nearby $(\lesssim 100 \mathrm{Mpc})$ SMBH and the later is applicable only to Type I (broad emission-line) AGNs, a trace of the population [46]. LISA could also constrain SMBH masses via equal-mass inspirals and EMRIs. However, it is only sensitive to mergers with masses $\lesssim 10^{7} M_{\odot}$ [47-49]. Our approach, on the other hand, probes SMBHs across almost the entire mass range to a distance of a few $\mathrm{Gpc}$ and applies independent of the SMBH being active or quiescent. It is thus an invaluable complementary to the existing methods. Furthermore, it also determines the outer orbit via measuring $a_{o}$ and $e_{o}$ that are hard to be extracted otherwise at $\mathcal{O}(\mathrm{Gpc})$ distances, thereby constraining the nuclei dynamics which currently has considerable theoretical uncertainties.

Hereafter, we will focus on the dS precession and how we can utilize it to measure $M_{3}$ and $a_{o}$. We neglect the subleading Lense-Thirring precession and Lidov-Kozai oscillations for simplicity (but see Ref. [50]) and treat both the inner and outer orbits as being circular (we will discuss the effects of eccentricities at the end of the Letter). Gaseous friction [11,51-55] and encounters with background objects $[5,56]$ have characteristic timescales ranging from thousands to millions of years and therefore can be ignored over an observation over $T_{\text {obs }} \simeq 5 \mathrm{yr}$ (see the Supplemental Material [41] for details). All the parameters in this Letter correspond to their inferred values in the detector frame [57]. We use geometrical units $G=c=1$. 


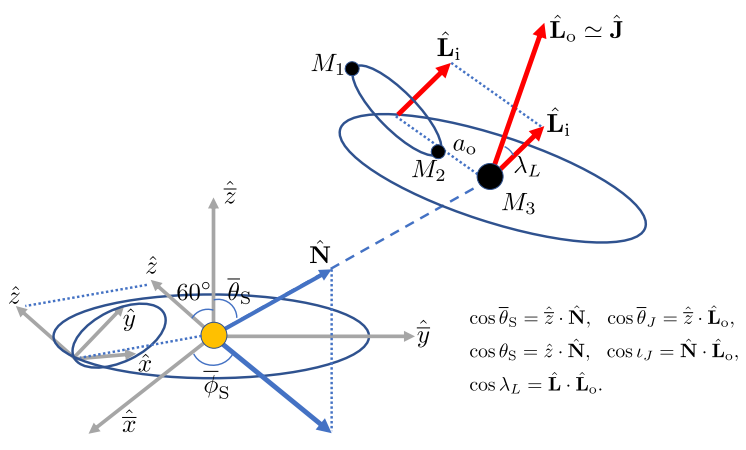

FIG. 2. Cartoon illustrating the geometry of the problem. Note the amplitudes of vectors are chosen only for visualization purpose.

Waveforms. - In Fig. 2 we demonstrate the geometry of the problem. We construct two reference frames. The $(x, y, z)$ frame is centered on the corner detector with $\hat{\boldsymbol{x}}$ and $\hat{\boldsymbol{y}}$ pointing along two arms of TianGO [29] (for LISA, this frame is constructed as in Ref. [58]). As the detector frame changes in both location and orientation, we also construct a fixed solar frame $(\bar{x}, \bar{y}, \bar{z})$ with $\hat{\bar{z}}$ perpendicular to the ecliptic. In the solar frame, the source's sky location $\hat{\boldsymbol{N}}$ and the total AM $\hat{\boldsymbol{J}}$ with $\boldsymbol{J} \equiv \boldsymbol{L}_{i}+\boldsymbol{L}_{o} \simeq \boldsymbol{L}_{o}$ are labeled with polar coordinates $\left(\bar{\theta}_{S}, \bar{\phi}_{S}\right)$ and $\left(\bar{\theta}_{J}, \bar{\phi}_{J}\right)$, respectively. We further define $l_{J}$ as the angle between $\hat{N}$ and $\hat{\boldsymbol{L}}_{o}$, and $\lambda_{L}$ the angle between $\hat{\boldsymbol{L}}_{i}$ and $\hat{\boldsymbol{L}}_{o}$. The problem now becomes projecting the GW radiation characterized by a time-varying orientation $\hat{\boldsymbol{L}}_{i}(t)$ onto an antenna with also time-varying coordinates $(\hat{\boldsymbol{x}}, \hat{\boldsymbol{y}}, \hat{z})$.

To obtain the antenna response of the GW detector, we follow Refs. [58,59]. The explicit expressions for various quantities can be found in the Supplemental Material [41]. The frequency-domain waveform under the stationaryphase approximation is

$$
\begin{aligned}
\tilde{h}(f)= & \Lambda(f) \tilde{h}_{C}(f)=\left[A_{+}^{2}(t) F_{+}^{2}(t)+A_{\times}^{2}(t) F_{\times}^{2}(t)\right]^{1 / 2} \\
& \times \exp \left\{-i\left[\Phi_{p}(t)+2 \Phi_{T}(t)+\Phi_{D}(t)\right]\right\} \tilde{h}_{C}(f),
\end{aligned}
$$

where $\Lambda$ characterizes the modulation due to antenna response and $\tilde{h}_{C}$ is the antenna-independent "carrier". We approximate $\tilde{h}_{C}$ with the quadrupole formula, including four intrinsic parameters, $\left(\mathcal{M}, D_{L}, t_{c}, \phi_{c}\right)$, corresponding to the chirp mass, luminosity distance, and time and phase of coalescence. The antenna pattern depends on time which is further a function of frequency, $t(f)=t_{c}-5(8 \pi f)^{-8 / 3} \mathcal{M}^{-5 / 3}$.

The changing orientations affect the amplitude both via $A_{+}=1+\left(\hat{\boldsymbol{L}}_{i} \cdot \hat{\boldsymbol{N}}\right)^{2}$ and $A_{\times}=-2 \hat{\boldsymbol{L}}_{i} \cdot \hat{\boldsymbol{N}}$, and via $F_{+(\times)}\left(\theta_{S}, \phi_{S}, \psi_{S}\right)$, where $\left(\theta_{S}, \phi_{S}\right)$ are the polar coordinates of $\boldsymbol{N}$ in the $(x, y, z)$ frame and $\psi_{S}$ is the polarization angle.

Besides amplitude modulations, there are also extra phase terms. The $\Phi_{p}$ term characterizes the polarization phase, and the precession of $\hat{\boldsymbol{L}}_{i}$ further gives rise to a Thomas precession term $\Phi_{T}$ [59]. Lastly, $\Phi_{D}$ describes a
Doppler phase due to motions of both the outer orbit and the detector orbiting around the Sun.

To this point the expressions are generic. A waveform is specified when one supplies information about the orbits (for $\left.\Phi_{D}\right)$ and the orientations $\hat{\boldsymbol{L}}_{i}$ and $(\hat{\boldsymbol{x}}, \hat{\boldsymbol{y}}, \hat{z})$.

We model the Doppler phase as [60]

$$
\begin{aligned}
\Phi_{D}= & 2 \pi f\left[a_{o} \sin \iota_{J} \cos \left(\Omega_{o} t-\phi^{(0)}\right)\right. \\
& \left.+\mathrm{AU} \sin \bar{\theta}_{S} \cos \left(2 \pi t / \mathrm{yr}-\bar{\phi}_{S}\right)\right],
\end{aligned}
$$

where $\phi^{(0)}$ characterizes an initial phase for the outer orbit. The dS precession of $\hat{\boldsymbol{L}}_{i}$ around $\hat{\boldsymbol{L}}_{o}$ can be written in terms of three additional parameters $\left(P_{\mathrm{dS}}, \lambda_{L}, \alpha_{0}\right)$ with $\alpha_{0}$ an initial phase characterizing the initial orientation of $\hat{\boldsymbol{L}}_{i}$. The detector's orientation for both LISA and TianGO is described in Ref. [62].

We compare in Fig. 3 sample waveforms with sensitivities of various space-borne detectors. The initial GW frequency $f^{(0)}$ is chosen such that the inner binary mergers in $5 \mathrm{yr}$, the fiducial value of $T_{\mathrm{obs}}$. For a stellar-mass inner binary (solid traces), various missions have similar sensitivities to the precession-induced modulation with the decihertz observatories having a greater total signal-tonoise ratio (SNR). With TianGO's sensitivity, the system corresponding to the purple-solid trace has a total SNR of 80 , and a SNR of 13 if we use only the data at least $0.1 \mathrm{yr}$ prior to the merger (i.e., integrating from the initial frequency to the dot markers). While the SNR from the final $0.1 \mathrm{yr}$ does not directly constrain the precession, it nonetheless reduces the uncertainties on other parameters that are partially degenerate with the precession signatures and is thus critical as well. Similarly, a joint detection of the source with ground-based detectors enhances the sensitivity

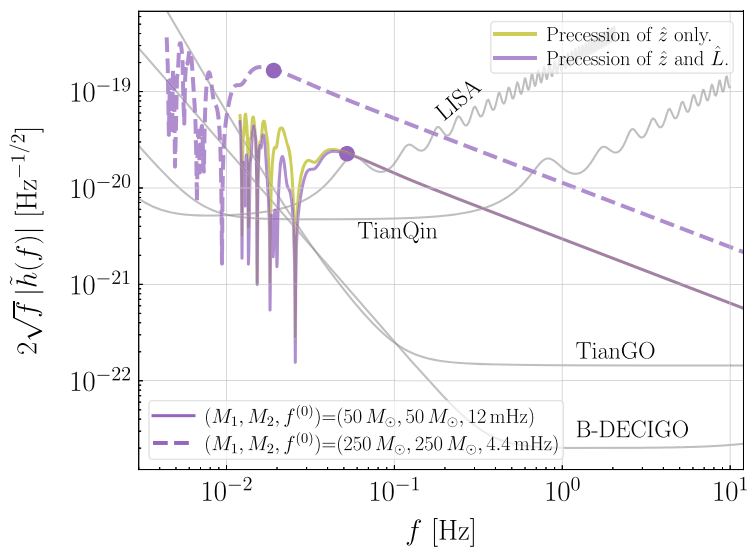

FIG. 3. Sample waveforms shown in $2 \sqrt{f}|\tilde{h}|$. The olive trace includes variation in the detector's orientation only, while the purple ones further include the $\mathrm{dS}$ precession. The initial frequency $f^{(0)}$ is chosen such that the binary merges in $T_{\text {obs }}=5 \mathrm{yr}$ and the dot symbols indicate the instant $0.1 \mathrm{yr}$ prior to the merger. We assumed $\left(D_{L}, \bar{\theta}_{S}, \bar{\phi}_{S}, \bar{\theta}_{J}, \bar{\phi}_{J}\right)=\left(1 \mathrm{Gpc}, 33^{\circ}, 147^{\circ}, 75^{\circ}, 150^{\circ}\right)$ and $\left(P_{\mathrm{dS}}, \lambda_{L}\right)=\left(2.7 \mathrm{yr}, 45^{\circ}\right)$. 


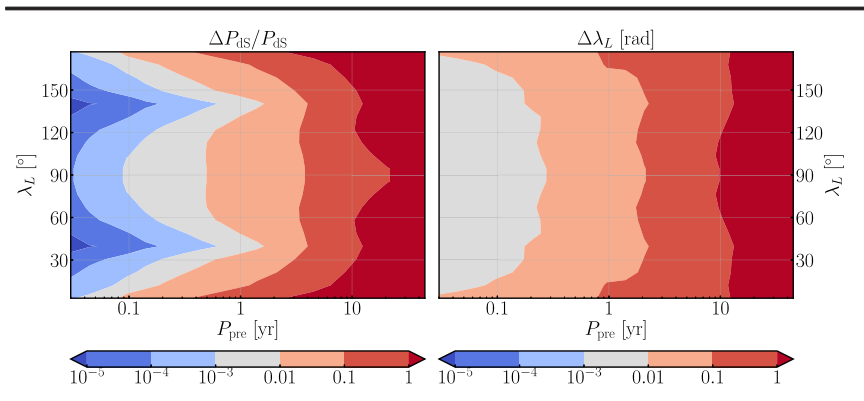

FIG. 4. PE results assuming a simple-precession problem (no Doppler phase of the outer orbit). We fix the inner binary to have $\left(M_{1}, M_{2}, f^{(0)}\right)=\left(50 M_{\odot}, 50 M_{\odot}, 12 \mathrm{mHz}\right)$. The source's sky location in the solar frame is $\left(D_{L}, \bar{\theta}_{S}, \bar{\phi}_{S}\right)=\left(1 \mathrm{Gpc}, 33^{\circ}, 147^{\circ}\right)$ and the orientation of the outer orbit is $\left(\bar{\theta}_{J}, \bar{\phi}_{J}\right)=\left(75^{\circ}, 150^{\circ}\right)$. Note the dS precession is detectable if $P_{\mathrm{dS}} \lesssim 10 \mathrm{yr}$ if the source is detected by TianGO alone.

further. If the inner binary consists of intermediate-mass BHs [the dashed trace; it has a total (early-stage) SNR of 36 (26) in LISA after combining two detectors' responses], then LISA alone would be able to detect the modulations.

Results.-We adopt the Fisher matrix formalism [58] to quantify the detectability [63]. We start by considering the parameter-estimation (PE) accuracy of a simple-precession problem (i.e., dropping the Doppler phase due to the outer orbit) and parameterize the modulation in terms of $\left(P_{\mathrm{dS}}, \lambda_{L}, \alpha_{0}\right)$. Our aim is to establish the detectability thresholds for $P_{\mathrm{dS}}$ and $\lambda_{L}$. The results are summarized in Fig. 4 (we have randomized $\alpha_{0}$ and plotted the median values). Throughout this section we assume the source is detected by TianGO [29] alone.

As expected, the accuracy in both $P_{\mathrm{dS}}$ and $\lambda_{L}$ improves as $P_{\mathrm{dS}}$ decreases, and at $P_{\mathrm{dS}} \simeq 2 T_{\mathrm{obs}}=10 \mathrm{yr}$ we have approximately $\Delta P_{\mathrm{dS}} / P_{\mathrm{dS}}<1$ and $\Delta \lambda_{L}<1 \mathrm{rad}$, marking the boundary of detectability.

Note that at $P_{\mathrm{dS}} \gtrsim 3 \mathrm{yr}$, the error $\Delta P_{\mathrm{dS}}$ is smallest when $\lambda_{L} \simeq 90^{\circ}$ as it maximizes the variation in the orientation. At smaller $P_{\mathrm{dS}}$, the optimal detectability is achieved at $\lambda_{L} \simeq 40^{\circ} \simeq l_{J}$ (and also at $140^{\circ}$ ). This is thanks to the Thomas phase $\Phi_{T}$. As shown in Ref. [59], when $\hat{N}$ is inside the precession cone $\left(\left|\hat{\boldsymbol{L}}_{o} \cdot \hat{\boldsymbol{L}}\right|<\left|\hat{\boldsymbol{L}}_{o} \cdot \hat{\boldsymbol{N}}\right|\right)$, each precession cycle the Thomas term contributes approximately $\left(-2 \pi \cos \lambda_{L}\right)$ to the phase. When $\hat{\boldsymbol{L}}_{o} \cdot \hat{\boldsymbol{L}}>\left|\hat{\boldsymbol{L}}_{o} \cdot \hat{\boldsymbol{N}}\right|$, however, the contribution per cycle changes sharply to about $2 \pi\left(-\cos \lambda_{L}+1\right)$ [65]. Consequently, when $\lambda_{L} \simeq l_{J}$ (or $\left.\pi-l_{J}\right), \Phi_{T}$ can be determined with high accuracy. Since the total $\Phi_{T}$ is proportional to the total number of precession cycles, it thus leads to good constraints on $P_{\mathrm{dS}}$.

As we know the detector's orbit, we do not see it significantly interfering with the results when $P_{\mathrm{dS}} \simeq 1 \mathrm{yr}$. Moreover, the Thomas phase is associated with the precession of $\hat{\boldsymbol{L}}_{i}$ only [58], further breaking the potential degeneracy between a changing $\hat{\boldsymbol{L}}$ and a changing $\hat{z}$. It is nonetheless crucial to include the detector's motion to constrain $\hat{N}[29,58]$.



FIG. 5. PE results combining both the dS precession and the Doppler phase shift. We assumed an opening angle between the inner and outer orbits of $\lambda_{L}=45^{\circ}$ and other parameters are the same as in Fig. 4. We also plotted the line of $P_{\mathrm{dS}}=10 \mathrm{yr}$. The grey regions have $\Delta \lambda_{L}>\lambda_{L}$ and therefore are excluded. Note $M_{3}$ can be constrained to $10 \%$ if $P_{\mathrm{dS}} \simeq 10 \mathrm{yr}$ with TianGO alone. The error decreases as $M_{3}$ increases thanks to the additional information provided by the amplitude of the Doppler phase.

We now combine the dS precession with the Doppler shift to study the constraints on the SMBH properties. We use $M_{3}$ and $a_{o}$ as free parameters and write $\Omega_{o}$ and $P_{\mathrm{dS}}$ in terms of $M_{3}$ and $a_{o}$. The initial phase $\phi^{(0)}$ is included and randomized over.

The result is shown in Fig. 5. We only include regions where $\Delta \lambda_{L} \leq \lambda_{L}$ so that the signature of precession is unambiguously detected. Note the boundary of $\Delta \lambda_{L}=\lambda_{L}$ is broadly consistent with the line of $P_{\mathrm{dS}}=10 \mathrm{yr}$, agreeing with the results we obtained in the simple-precession analysis. Along the line of $P_{\mathrm{dS}}=10 \mathrm{yr}$, the fractional error in the SMBH mass is constrained to $\Delta M_{3} / M_{3} \sim 10 \%$, demonstrating a direct determination of the SMBH property is indeed possible. We further find that $\Delta \log a_{o} \simeq$ $\Delta \log M_{3} / 3$ for most of the parameter spaces because $\Omega_{o}$ is determined with the highest accuracy among all the parameters describing the modulations.

Along the line of constant $P_{\mathrm{dS}}$, the error decreases with increasing $M_{3}$. This is because the "modulation depth" on the Doppler phase [Eq. (3)] increases with $M_{3}$. With the Doppler shift alone, we cannot utilize the modulation depth due to the unknown $\sin l_{J}$. Once the precession is included, however, $\hat{\boldsymbol{L}}_{o}$ serves as the precession axis of $\hat{\boldsymbol{L}}_{i}$, allowing the outer orbit's inclination to be inferred. Once we know $\sin l_{J}$, the modulation depth provides another measurement of $a_{o}$, enhancing the sensitivity further.

Summary and discussion.-Our analysis so far has considered detections by TianGO alone. As ground-based detectors are more sensitive to stellar-mass BHs [29], they could constrain intrinsic parameters with much higher accuracy. We thus estimate the joint-detection effect by still computing the Fisher matrix using a spaceborne detector's sensitivity but treating $\left(\mathcal{M}, \phi_{c}, t_{c}\right)$ as known parameters. For a system with $\left(M_{3}, a_{o}\right)=$ $\left(10^{8} M_{\odot}, 100 M_{3}\right)$ and the rest the same as in Fig. 5, the errors in $\left(M_{3}, a_{o}\right)$ can be dramatically improved to $\Delta \log M_{3}=1.7 \times 10^{-4}\left(5.2 \times 10^{-2}\right) \simeq 3 \log a_{o}$ assuming 
the sensitivity of TianGO (LISA). The uncertainty in $\lambda_{L}$ is also reduced by about a factor of 6 to $\Delta \lambda_{L}=0.02 \mathrm{rad}$ for both TianGO and LISA. If a source instead has $a_{o}=300 M_{3}$ with $P_{\text {pre }} \simeq 100 \mathrm{yr}$, we find a median error $\Delta \lambda_{L}=0.72 \mathrm{rad}<\lambda_{L}$ with LISA's sensitivity after randomizing initial phases, indicating the precession would still be detectable. Knowing the source's distance and sky location further improves the accuracy in $\lambda_{L}$ by a factor of a few. For $a_{o}=300 M_{3}$ and LISA's sensitivity, we find $\Delta \lambda_{L}=0.16 \mathrm{rad}$ in this case.

We assumed both circular inner and outer orbits. In reality, finite eccentricities are expected especially if the inner binary is formed via dynamical channels. One plausible scenario is that both $e_{i}$ and $e_{o}$ follow a thermal distribution, with $e_{i, o}^{2}$ uniform in $[0,1)[4,67]$.

An elliptic outer orbit enhances the detectability. Note $e_{0}$ does not affect the inference accuracy of $\Omega_{o}$ and itself can be well constrained from the Doppler shift (as demonstrated in the Supplemental Material [41]). Although the instantaneous precession rate [68] should be used for waveform modeling, the secular version [Eq. (1)] nonetheless indicates the qualitative effect of $e_{o}$, which is to make the rate greater by a factor of $1 /\left(1-e_{o}^{2}\right)$. Thus at a fixed $a_{o}$ the waveform is modulated by more precession cycles, making its signature more prominent. It also allows a system at greater $a_{o}$ to potentially experience a significant modulation (lower panel of Fig. 1).

The eccentricity of the inner orbit $e_{i}$ modifies only the carrier $\tilde{h}_{c}$. Therefore, it affects the results mostly through affecting the overall SNR. Following Ref. [69], for mild eccentricities $\left(e_{i} \leq 0.7\right.$ at $\left.a_{i}=1.4 \times 10^{-3} \mathrm{AU}\right)$, we find both the total SNR and that from the early stage $(\geq 0.1 \mathrm{yr}$ prior to merger) in fact increase for TianGO, and decrease by a small amount (factor of 3 ) for LISA. A more extreme eccentricity would make the inner orbit decay too quickly if we fix the initial $a_{i}^{(0)}$. Nonetheless, such a system can merge within $T_{\text {obs }}$ starting at much greater initial separations of $\mathcal{O}(0.1)$ AU. From the evolution from 0.1 AU to $10^{-3}$ AU we can still obtain an integration time of more than a year and a SNR of about 5 (with the sensitivity of TianGO). Therefore, our results should not change qualitatively by the inner eccentricity (detailed calculations presented in the Supplemental Material [41]).

We did not include the precessions of $\hat{\boldsymbol{L}}_{i}$ due to the spins of $M_{1(2)}$. Nevertheless, this should be well distinguishable from the precessions around $\hat{\boldsymbol{L}}_{o}$ thanks to the separation in scales. The spin-induced opening angle is $\lesssim M_{1}^{2} / L_{i} \sim 1^{\circ}$ when $f \sim 0.01 \mathrm{~Hz}$, in general much smaller than $\Lambda_{L}$ which distributes approximately uniformly between $0^{\circ}$ and $180^{\circ}$ (e.g., Ref. [70]). Moreover, the spin-induced precession rate is $\sim L_{i} / a_{i}^{3}$ [59], corresponding to a period of 10 days when $f=0.01 \mathrm{~Hz}$, and the period decreases further as the inner binary decays. In contrast, the $\mathrm{dS}$ precession around $L_{o}$ has a constant and much longer period.
Whereas we used the quadrupole formula for the carrier, our formalism can be readily extended to incorporated more complicated dynamics of the inner binary (higher-order relativistic corrections as well as environmental effects due to gas [54] and/or gravitational lensing $[71,72]$ that alter the observed chirp mass [73]) by replacing the carrier part with the appropriate $\tilde{h}_{C}(f)$. Similar to the inner eccentricity, changing the carrier affects the detectability of extrinsic modulations mostly through changing the overall SNR.

To conclude, we demonstrated that the dS precession of $\hat{\boldsymbol{L}}_{i}$ around $\hat{\boldsymbol{L}}_{o}$ is detectable. The detectability threshold is $P_{\mathrm{dS}} \simeq 10 \mathrm{yr}$ with space-borne detectors alone and $P_{\mathrm{dS}} \simeq 100 \mathrm{yr}$ if the source is jointly detected by groundbased detectors. This effect allows a direct determination of the SMBH mass to better than $10 \%$ at Gpc distances and applies to both active and quiescent SMBHs. It also constrains the dynamics in galactic nuclei by pinpointing the outer orbit. Future studies incorporating the orbital eccentricities and subleading effects, as well as extending the PE to a more rigorous Bayesian framework would be of great value.

We are thankful for the helpful comments from Imre Bartos, Karan Jani, and the referees during the preparation of this work. We are grateful to Hiroyuki Nakano for kindly providing us the sensitivity curve of B-DECIGO. H. Y. acknowledges the support by the Sherman Fairchild Foundation. Y.C. is supported by the Simons Foundation (Grant No. 568762), and the National Science Foundation, through Grants No. PHY-2011961, No. PHY-2011968, and No. PHY-1836809. The authors also gratefully acknowledge the computational resources provided by the LIGO Laboratory and support by NSF Grants No. PHY-0757058 and No. PHY-0823459.

"hangyu@ caltech.edu

[1] LIGO Scientific Collaboration, Advanced LIGO, Classical Quantum Gravity 32, 074001 (2015).

[2] F. Acernese, M. Agathos, K. Agatsuma, D. Aisa, N. Allemandou, A. Allocca, J. Amarni, P. Astone et al., Advanced Virgo: A second-generation interferometric gravitational wave detector, Classical Quantum Gravity 32, 024001 (2015).

[3] R. M. O'Leary, B. Kocsis, and A. Loeb, Gravitational waves from scattering of stellar-mass black holes in galactic nuclei, Mon. Not. R. Astron. Soc. 395, 2127 (2009).

[4] F. Antonini and H. B. Perets, Secular Evolution of Compact Binaries near Massive Black Holes: Gravitational Wave Sources and Other Exotica, Astrophys. J. 757, 27 (2012).

[5] F. Antonini and F. A. Rasio, Merging black hole binaries in galactic nuclei: Implications for Advanced-LIGO detections, Astrophys. J. 831, 187 (2016).

[6] C. Petrovich and F. Antonini, Greatly enhanced merger rates of compact-object binaries in non-spherical nuclear star clusters, Astrophys. J. 846, 146 (2017). 
[7] N. W. C. Leigh, A. M. Geller, B. McKernan, K. E. S. Ford, M. M. Mac Low, J. Bellovary, Z. Haiman, W. Lyra, J. Samsing, M. O'Dowd, B. Kocsis, and S. Endlich, On the rate of black hole binary mergers in galactic nuclei due to dynamical hardening, Mon. Not. R. Astron. Soc. 474, 5672 (2018).

[8] X. Chen and W.-B. Han, Extreme-mass-ratio inspirals produced by tidal capture of binary black holes, Commun. Phys. 1, 53 (2018).

[9] G. Fragione, N. W. C. Leigh, and R. Perna, Black hole and neutron star mergers in galactic nuclei: The role of triples, Mon. Not. R. Astron. Soc. 488, 2825 (2019).

[10] B. McKernan, K. E. S. Ford, W. Lyra, and H. B. Perets, Intermediate mass black holes in AGN discs-I. Production and growth, Mon. Not. R. Astron. Soc. 425, 460 (2012).

[11] I. Bartos, B. Kocsis, Z. Haiman, and S. Márka, Rapid and bright stellar-mass binary black hole mergers in active galactic nuclei, Astrophys. J. 835, 165 (2017).

[12] N. C. Stone, B. D. Metzger, and Z. Haiman, Assisted inspirals of stellar mass black holes embedded in AGN discs: Solving the 'final au problem', Mon. Not. R. Astron. Soc. 464, 946 (2017).

[13] B. McKernan, K. E. S. Ford, J. Bellovary, N. W. C. Leigh, Z. Haiman, B. Kocsis, W. Lyra, M. M. Mac Low, B. Metzger, M. O'Dowd, S. Endlich, and D. J. Rosen, Constraining stellar-mass black hole mergers in AGN disks detectable with LIGO, Astrophys. J. 866, 66 (2018).

[14] H. Tagawa, Z. Haiman, and B. Kocsis, Formation and evolution of compact object binaries in AGN disks, arXiv:1912.08218.

[15] B. McKernan, K. E. S. Ford, I. Bartos, M. J. Graham, W. Lyra, S. Marka, Z. Marka, N. P. Ross, D. Stern, and Y. Yang, Ram-pressure stripping of a kicked hill sphere: Prompt electromagnetic emission from the merger of stellar mass black holes in an AGN accretion disk, Astrophys. J. 884, L50 (2019).

[16] Y. Yang, I. Bartos, V. Gayathri, K. E. S. Ford, Z. Haiman, S. Klimenko, B. Kocsis, S. Márka, Z. Márka, B. McKernan, and R. O'Shaughnessy, Hierarchical Black Hole Mergers in Active Galactic Nuclei, Phys. Rev. Lett. 123, 181101 (2019).

[17] A. Secunda, J. Bellovary, M.-M. Mac Low, K. E. S. Ford, B. McKernan, N. W. C. Leigh, W. Lyra, and Z. Sándor, Orbital migration of interacting stellar mass black holes in disks around supermassive black holes, Astrophys. J. 878, 85 (2019).

[18] E. C. Bellm, S. R. Kulkarni, M. J. Graham, R. Dekany, R. M. Smith, R. Riddle, F. J. Masci, G. Helou et al., The Zwicky transient facility: System overview, performance, and first results, Publ. Astron. Soc. Pac. 131, 018002 (2019).

[19] M. J. Graham, S. R. Kulkarni, E. C. Bellm, S. M. Adams, C. Barbarino, N. Blagorodnova, D. Bodewits, B. Bolin et al., The Zwicky transient facility: Science objectives, Publ. Astron. Soc. Pac. 131, 078001 (2019).

[20] M. J. Graham, K. E. S. Ford, B. McKernan, N. P. Ross, D. Stern, K. Burdge, M. Coughlin, S. G. Djorgovski et al., Candidate Electromagnetic Counterpart to the Binary Black Hole Merger Gravitational-Wave Event s190521g, Phys. Rev. Lett. 124, 251102 (2020).

[21] R. Abbott, T. D. Abbott, S. Abraham, F. Acernese, K. Ackley, C. Adams, R. X. Adhikari, V. B. Adya et al. (LIGO Scientific and Virgo Collaborations), GW190521: A Binary
Black Hole Merger with a Total Mass of $150 M_{\odot}$, Phys. Rev. Lett. 125, 101102 (2020).

[22] R. Abbott, T. D. Abbott, S. Abraham, F. Acernese, K. Ackley, C. Adams, R. X. Adhikari, V. B. Adya et al., Properties and astrophysical implications of the $150 \mathrm{~m} \odot$ binary black hole merger GW190521, Astrophys. J. 900, L13 (2020).

[23] P. Amaro-Seoane, H. Audley, S. Babak, J. Baker, E. Barausse, P. Bender, E. Berti, P. Binetruy et al., Laser interferometer space antenna, arXiv:1702.00786.

[24] J. Luo, L.-S. Chen, H.-Z. Duan, Y.-G. Gong, S. Hu, J. Ji, Q. Liu, J. Mei, V. Milyukov, M. Sazhin, C.-G. Shao, V. T. Toth, H.-B. Tu, Y. Wang, Y. Wang, H.-C. Yeh, M.-S. Zhan, Y. Zhang, V. Zharov, and Z.-B. Zhou, TianQin: A space-borne gravitational wave detector, Classical Quantum Gravity 33, 035010 (2016).

[25] W.-R. Hu and Y.-L. Wu, The Taiji program in space for gravitational wave physics and the nature of gravity, Natl. Sci. Rev. 4, 685 (2017).

[26] T. Nakamura, M. Ando, T. Kinugawa, H. Nakano, K. Eda, S. Sato, M. Musha, T. Akutsu, T. Tanaka, N. Seto, N. Kanda, and Y. Itoh, Pre-DECIGO can get the smoking gun to decide the astrophysical or cosmological origin of GW150914-like binary black holes, Prog. Theor. Exp. Phys. 2016, 093E01 (2016).

[27] S. Kawamura, M. Ando, N. Seto, S. Sato, M. Musha, I. Kawano, J. Yokoyama, T. Tanaka et al., Current status of space gravitational wave antenna DECIGO and B-DECIGO, arXiv:2006.13545.

[28] M. Arca Sedda, C. P. L. Berry, K. Jani, P. Amaro-Seoane, P. Auclair, J. Baird, T. Baker, E. Berti et al., The missing link in gravitational-wave astronomy: Discoveries waiting in the decihertz range, Classical Quantum Gravity 37, 215011 (2020).

[29] K. A. Kuns, H. Yu, Y. Chen, and R. X. Adhikari, Astrophysics and cosmology with a decihertz gravitational-wave detector: Tiango, Phys. Rev. D 102, 043001 (2020).

[30] T. Damour and J. H. Taylor, Strong-field tests of relativistic gravity and binary pulsars, Phys. Rev. D 45, 1840 (1992).

[31] P. Amaro-Seoane, Relativistic dynamics and extreme mass ratio inspirals, Living Rev. Relativity 21, 4 (2018).

[32] P. Amaro-Seoane, Extremely large mass-ratio inspirals, Phys. Rev. D 99, 123025 (2019).

[33] W.-B. Han and X. Chen, Testing general relativity using binary extreme-mass-ratio inspirals, Mon. Not. R. Astron. Soc. 485, L29 (2019).

[34] W.-B. Han, X.-Y. Zhong, X. Chen, and S. Xin, Very extreme mass-ratio bursts in the Galaxy and neighbouring galaxies in relation to space-borne detectors, Mon. Not. R. Astron. Soc. 498, L61 (2020).

[35] For future convenience, we will refer to the orbit of the stellar-mass BH binary as the inner orbit, and its center of mass orbiting the SMBH as the outer orbit.

[36] K. Inayoshi, N. Tamanini, C. Caprini, and Z. Haiman, Probing stellar binary black hole formation in galactic nuclei via the imprint of their center of mass acceleration on their gravitational wave signal, Phys. Rev. D 96, 063014 (2017). 
[37] L. Randall and Z.-Z. Xianyu, A direct probe of mass density near inspiraling binary black holes, Astrophys. J. 878, 75 (2019).

[38] C. M. Will, New General Relativistic Contribution to Mercury's Perihelion Advance, Phys. Rev. Lett. 120, 191101 (2018).

[39] B. Liu, D. Lai, and Y.-H. Wang, Binary mergers near a supermassive black hole: Relativistic effects in triples, Astrophys. J. 883, L7 (2019).

[40] H. Yu, S. Ma, M. Giesler, and Y. Chen, Spin and eccentricity evolution in triple systems: From the Lidov-Kozai interaction to the final merger of the inner binary, Phys. Rev. D 102, 123009 (2020).

[41] See Supplemental Material at http://link.aps.org/ supplemental/10.1103/PhysRevLett.126.021101 for explicit expressions of timescales and the GW waveform, as well as discussions on the effects of the outer and inner eccentricities.

[42] L. G. Kiseleva, S. J. Aarseth, P. P. Eggleton, and R. de La Fuente Marcos, Formation and evolution of hierarchical triple systems in open clusters, in The Origins, Evolution, and Destinies of Binary Stars in Clusters, Astronomical Society of the Pacific Conference Series, Vol. 90, edited by E. F. Milone and J. C. Mermilliod (Astronomical Society of the Pacific, San Francisco, 1996), p. 433.

[43] J. M. Bellovary, M.-M. Mac Low, B. McKernan, and K. E. S. Ford, Migration traps in disks around supermassive black holes, Astrophys. J. 819, L17 (2016).

[44] J. H. VanLandingham, M. C. Miller, D. P. Hamilton, and D. C. Richardson, The role of the Kozai-Lidov mechanism in black hole binary mergers in galactic centers, Astrophys. J. 828, 77 (2016).

[45] É. É. Flanagan and S. A. Hughes, Measuring gravitational waves from binary black hole coalescences. I. Signal to noise for inspiral, merger, and ringdown, Phys. Rev. D 57, 4535 (1998).

[46] B. M. Peterson, Measuring the masses of supermassive black holes, Space Sci. Rev. 183, 253 (2014).

[47] S. A. Hughes, Untangling the merger history of massive black holes with LISA, Mon. Not. R. Astron. Soc. 331, 805 (2002).

[48] J. R. Gair, L. Barack, T. Creighton, C. Cutler, S. L. Larson, E. S. Phinney, and M. Vallisneri, Event rate estimates for LISA extreme mass ratio capture sources, Classical Quantum Gravity 21, S1595 (2004).

[49] J. R. Gair, S. Babak, A. Sesana, P. Amaro-Seoane, E. Barausse, C. P. L. Berry, E. Berti, and C. Sopuerta, Prospects for observing extreme-mass-ratio inspirals with LISA, J. Phys. Conf. Ser. 840, 012021 (2017).

[50] Y. Fang, X. Chen, and Q.-G. Huang, Impact of a spinning supermassive black hole on the orbit and gravitational waves of a nearby compact binary, Astrophys. J. 887, 210 (2019).

[51] E. C. Ostriker, Dynamical friction in a gaseous medium, Astrophys. J. 513, 252 (1999).

[52] C. Baruteau, J. Cuadra, and D. N. C. Lin, Binaries migrating in a gaseous disk: Where are the galactic center binaries?, Astrophys. J. 726, 28 (2011).

[53] A. Antoni, M. MacLeod, and E. Ramirez-Ruiz, The evolution of binaries in a gaseous medium: Three- dimensional simulations of binary Bondi-Hoyle-Lyttleton accretion, Astrophys. J. 884, 22 (2019).

[54] X. Chen, Z.-Y. Xuan, and P. Peng, Fake massive black holes in the milli-hertz gravitational-wave band, Astrophys. J. 896, 171 (2020).

[55] A. Derdzinski, D. D’Orazio, P. Duffell, Z. Haiman, and A. Macfadyen, Evolution of gas disc-embedded intermediate mass ratio inspirals in the LISA band, arXiv:2005 .11333 .

[56] K. Gültekin, M. C. Miller, and D. P. Hamilton, Growth of intermediate-mass black holes in globular clusters, Astrophys. J. 616, 221 (2004).

[57] One can show that from the source frame to the detector frame, $M_{3} \rightarrow(1+z) M_{3}$ and $a_{0} \rightarrow(1+z) a_{0}$ due to a constant redshift $z$. Note $z$ may include both the cosmological redshift $(\sim 0.2$ at $1 \mathrm{GPc})$ and that due to the gravitational potential of $M_{3}(\lesssim 0.01$ for typical sources at $a_{\mathrm{o}} \gtrsim 100 M_{3}$ ).

[58] C. Cutler, Angular resolution of the LISA gravitational wave detector, Phys. Rev. D 57, 7089 (1998).

[59] T. A. Apostolatos, C. Cutler, G. J. Sussman, and K. S. Thorne, Spin-induced orbital precession and its modulation of the gravitational waveforms from merging binaries, Phys. Rev. D 49, 6274 (1994).

[60] Here we have dropped the Shapiro time delay for simplicity, which contributes an extra phase $2 \pi t_{S}$ with $t_{S}=$ $2 M_{3} \log \left[1 /\left(1-\sin t_{J} \sin \phi_{\mathrm{o}}\right)\right]$ [61]. For most $l_{J} \neq 90^{\circ}$, $t_{S} \sim 2 M_{3} \ll a_{\mathrm{o}}$.

[61] R. Blandford and S. A. Teukolsky, Arrival-time analysis for a pulsar in a binary system, Astrophys. J. 205, 580 (1976).

[62] S. V. Dhurandhar, K. R. Nayak, S. Koshti, and J. Y. Vinet, Fundamentals of the LISA stable flight formation, Classical Quantum Gravity 22, 481 (2005).

[63] As a caveat, we note the Fisher matrix may be inaccurate when the SNR is low [64]. Therefore, future studies consider this problem in a full Bayesian framework would be of great value.

[64] M. Vallisneri, Use and abuse of the Fisher information matrix in the assessment of gravitational-wave parameterestimation prospects, Phys. Rev. D 77, 042001 (2008).

[65] See Sec. IV of Ref. [66] for a treatment that gives the same waveforms without introducing this apparently discontinuous Thomas Precession phase.

[66] A. Buonanno, Y. Chen, and M. Vallisneri, Detecting gravitational waves from precessing binaries of spinning compact objects: Adiabatic limit, Phys. Rev. D 67, 104025 (2003).

[67] D. C. Heggie, Binary evolution in stellar dynamics., Mon. Not. R. Astron. Soc. 173, 729 (1975).

[68] B. M. Barker and R. F. O'Connell, Gravitational two-body problem with arbitrary masses, spins, and quadrupole moments, Phys. Rev. D 12, 329 (1975).

[69] L. Barack and C. Cutler, LISA capture sources: Approximate waveforms, signal-to-noise ratios, and parameter estimation accuracy, Phys. Rev. D 69, 082005 (2004).

[70] H. Tagawa, Z. Haiman, I. Bartos, and B. Kocsis, Spin evolution of stellar-mass black hole binaries in active galactic nuclei, Astrophys. J. 899, 26 (2020). 
[71] B. Kocsis, High-frequency gravitational waves from supermassive black holes: Prospects for LIGO-VIRGO detections, Astrophys. J. 763, 122 (2013).

[72] X. Chen, S. Li, and Z. Cao, Mass-redshift degeneracy for the gravitational-wave sources in the vicinity of supermassive black holes, Mon. Not. R. Astron. Soc. 485, L141 (2019).

[73] X. Chen, Distortion of gravitational-wave signals by astrophysical environments, arXiv:2009.07626. 